\title{
The ability of external immobilizers to restrict movement of the cervical spine: a systematic review
}

\author{
Micha Holla $^{1}$ - Joske M. R. Huisman ${ }^{1}$ - Nico Verdonschot ${ }^{2,3} \cdot$ Jon Goosen $^{4}$ • \\ Allard J. F. Hosman ${ }^{1}$ Gerjon Hannink ${ }^{2}$
}

Received: 22 May 2015/Revised: 7 January 2016/Accepted: 7 January 2016/Published online: 31 March 2016

(c) The Author(s) 2016. This article is published with open access at Springerlink.com

\begin{abstract}
Purpose To review the ability of various types of external immobilizers to restrict cervical spine movement.

Methods With a systematical review of original scientific articles, data on range of motion, type of used external immobilization device and risk of bias were extracted. The described external immobilization devices were grouped and the mean restriction percentage and standard deviation were calculated. Finally, each device was classified based on its ability to restrict movement of the cervical spine, according to five levels of immobilization: poor (MIL $<20 \%$ ), fair (MIL 20-40\%), moderate (MIL 40-60\%), substantial (MIL 60-80\%), and nearly complete (MIL $\geq 80 \%$ ).

Results The ability to reduce the range of motion by soft collars was poor in all directions. The ability of cervicohigh thoracic devices was moderate for flexion/extension but poor for lateral bending and rotation. The ability of cervico-low thoracic devices to restrict flexion/extension and rotation was moderate, while their ability to restrict lateral bending was poor. All cranio-thoracic devices for non-ambulatory patients restricted cervical spine
\end{abstract}

Micha Holla

Micha.Holla@radboudumc.nl

1 Spine Unit, Department of Orthopedics, Radboud University Medical Center, Geert Grooteplein Zuid 10, 6500 HB Nijmegen, Gelderland, The Netherlands

2 Orthopedic Research Lab, Department of Orthopedics, Radboud University Medical Center, Nijmegen, The Netherlands

3 Laboratory for Biomechanical Engineering, University of Twente, Enschede, The Netherlands

4 Sint Maartenskliniek, Nijmegen, The Netherlands movement substantial in all directions. The ability of vests with non-invasive skull fixation was substantial in all directions. No studies with healthy adults were identified with respect to cranial traction and halo vests with skull pins and their ability to restrict cervical movement.

Conclusions Soft collars have a poor ability to reduce mobility of the cervical spine. Cervico-high thoracic devices primarily reduce flexion and extension, but they reduce lateral bending and rotation to a lesser degree. Cervico-low thoracic devices restrict lateral bending to the same extent as cervico-high thoracic devices, but are considerably more effective at restricting flexion, extension, and rotation. Finally, cranio-thoracic devices nearly fully restrict movement of the cervical spine.

Keywords Systematic review - Cervical spine . Immobilization · Movement · Orthotic devices

\section{Introduction}

Worldwide, hundreds of patients receive external immobilization of the cervical spine each day, and this intervention is believed to have high clinical significance [1]. In the United States alone, each year five million patients receive some form of spinal immobilization [2].

Several methods to externally immobilize the cervical spine are currently available and are based on immobilizing specific parts of the body. The Advanced Trauma Life Support foundation recommends immobilizing all patients with potential cervical spine injury using a rigid collar, head blocks, and spine board. However, there is currently insufficient evidence to support this guideline [3].

To date, no properly designed randomized controlled trial has compared the various methods of spinal 
immobilization with respect to their ability to reduce mortality, prevent neurological disability, increase spinal stability, and minimize adverse effects in trauma patients [4]. Before clinically relevant studies of various treatment strategies can be reported, consensus is needed regarding the definition of currently available immobilizers and their ability to restrict cervical movement.

Previously published systematic reviews of the ability of immobilizing devices to restrict cervical movement specifically addressed individual types of collars and orthotic devices [5]. However, to date, no study has systematically reviewed all available types of devices designed to restrict cervical movement (e.g., cranial traction, spine boards, Minerva casts, halo vests, etc.).

One reason for this lack of systematic reviews may be the historical absence of a validated system for classifying this wide range of external cervical devices [6]. Recently, however, a validated classification system to define and compare various types of external cervical immobilizers was published [7].

The objective of this study was to systematically review all articles published regarding external cervical immobilizers and to quantify and compare their ability to restrict movement of the cervical spine.

\section{Materials and methods}

\section{Database search}

A literature search was performed in accordance with the 2009 Method Guidelines for Systematic Reviews in the Cochrane Back Review Group (CBRG) [8]. The electronic databases MEDLINE, EMBASE, the Cochrane Central Register of Controlled Trials (CENTRAL), and the CBRG trials register were searched by one reviewer (author J. H.) to identify all studies regarding external immobilizers and their ability to restrict movement of the cervical spine. All databases were searched from their inception through August 1, 2012. References from relevant research articles and systematic reviews were scanned and used to identify additional studies. The search strategy is presented in detail in Appendix.

\section{Criteria for eligibility and selection of articles}

After duplicate articles were removed, all articles identified from the database search were screened for eligibility based on the title and abstract. The eligibility criteria were established by two reviewers (authors J. H. and M. H.), who combined the objective of this study with the Crag's guidelines for systematic reviews [8].
Only studies that reported the reduction in cervical motion in at least one of three planes (sagittal for flexion and extension; coronal for lateral bending; and axial for rotation) were included. Articles written in English, German and Latin based languages were included. Articles in any other languages were excluded. Studies that only reported the reduction in intervertebral distance in millimeters were excluded. Only studies performed in healthy adults (and/or human cadavers) with no history of spinal pathology were included, and only studies that reported the reduction in cervical motion compared with that subject's normal motion were included. Only studies that used a reliable and reproducible measuring method as described by Williams et al. [9] (e.g., electro-magnetic field, 3M optical-electrical devices, digital dual inclinometers, goniometers, or conventional radiography) were included. Studies that relied solely on a visual estimation for determining restricted movement were excluded. Finally, studies that reported only the mean reduction in motion rather than individual results were excluded.

\section{Quality assessment of included articles}

Full-text versions of all included articles were downloaded and assessed for potential bias by two reviewers (authors M. H. and J. G.), who applied the Quality Assessment Tool for Quantitative Studies (EPHPP) [8]. Selected studies were rated strong/moderate/weak for the following components: selection bias, study design, confounders, blinding, data collection methods, withdrawals and dropouts. Studies with three or more strong ratings and without any weak rating were considered to be studies of good quality. Studies rated with two or more weak ratings were considered low quality studies. Other studies were rated moderate. Low or moderate quality studies were marked with an asterisk in the tables and figures; these studies were excluded from our conclusions. One of the review authors (MH) was also an author of one of the included articles and was excluded from any decision making regarding this article.

\section{Data extraction}

The following data were extracted from the included articles: first author's surname, year of publication, type and number of participants, name of external immobilizer studied, and mean range of motion with standard deviation and/or $95 \%$ confidence interval. If data were not available in the article's text or tables, the results were extrapolated from the graphs. If standard deviation was not reported, it was calculated from the $95 \%$ confidence interval [10]. If the percentages of unrestricted motion for lateral bending 
and/or rotation were reported separately for the right and left sides, the mean and standard deviation were calculated using the mean of the variances [11].

All immobilizers described in the selected articles were classified independently by two reviewers (JH and $\mathrm{MH}$ ) in accordance with a validated classification system [7]. This system is based on the anatomical region (or regions) that the device supports and includes the following five main types (see Fig. 1): A, cervical devices; B, cervico-thoracic devices; $\mathrm{C}$, cranial traction; $\mathrm{D}$, cranio-thoracic devices for non-ambulatory patients; and E, cranio-thoracic devices for ambulatory patients.

For all immobilizers analyzed, a mean restriction percentage (MRP) was calculated. First, we obtained the difference in the reported cervical range of motion with and without the immobilizer; this difference was then divided by the cervical range of motion without the immobilizer. In clinical practice, patients with cervical spine injury, a certain safety margin must be applied. Therefore, a minimal immobilization limit (MIL) was introduced. The MIL was calculated by subtracting one standard deviation from the MRP. Finally, to classify the ability of each external immobilizer to restrict cervical mobility, we defined the following five levels of immobilization: poor (MIL $<20 \%$ ), fair (MIL 20-40\%), moderate (MIL 40-60\%), substantial (MIL 60-80\%), and nearly complete (MIL $\geq 80 \%$ ).

\section{Results}

\section{Database search results and included articles}

Our database search yielded 2272 records plus six additional records from the references therein. After removing 99 duplicates, the total number of potentially eligible articles was 2179. After screening the abstracts and titles, 2131 articles were excluded. Three records were excluded due to the language of the text (Hebrew, Russian, and Slovak). Forty-eight full-text articles were retrieved for further analysis, ten of which were subsequently excluded because they did not report standard deviations or $95 \%$ confidence intervals.

An additional 25 full-text articles were excluded because the reduction in motion was reported as the mean for the entire cohort, and MRP could be calculated for these studies. Thus, 13 biomechanical studies investigating 23 different cervical immobilization devices in healthy adult volunteers were included in the final analysis. Figure 2 provides a flowchart depicting the inclusion and exclusion of articles used in this systematic review.

\section{Quality assessment}

The results of quality assessment of all included studies are presented in Table 1. Three of the 13 studies were

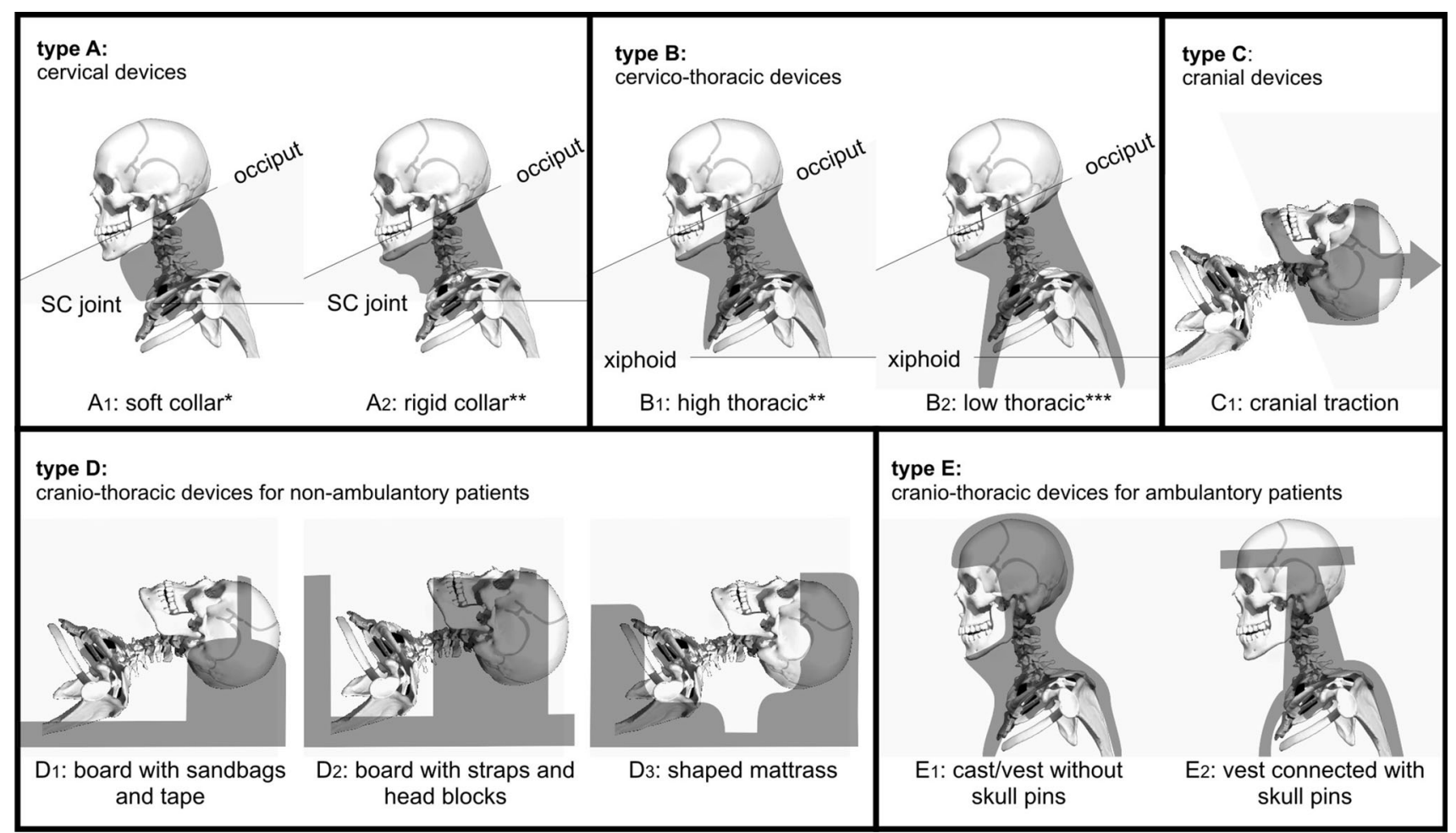

Fig. 1 Classification system for external cervical immobilizing devices based on the anatomical regions in which these devices provide support 
rated as a study of moderate quality. The study by Gavin et al. [12] excluded seven of their 20 subjects because of poor fluoroscopy image quality. Their reason for excluding these subjects was related to the shape and movement of the cervical spine and therefore represents a potential bias. Hammacher et al. [13] tested each immobilization device on a small number of participants and found major differences in MRP between left and right rotation for all immobilization devices. In some cases, their reported standard deviation was larger than the mean value.

Johnson et al. [17] tested six different immobilizers. Three immobilizers were applied to each subject without any further clarification. As randomization was not described and age and gender were not evenly distributed in different immobilizers, this study was considered to have potential selection bias and/or confounding. Because these three studies met our inclusion criteria, their results are included in the tables and figures (marked with an asterisk); however, their outcomes were excluded from our analysis and final conclusions. Due to the relatively low number of relevant studies and the wide variation in their methods, no meta-analysis was performed.

\section{Types of immobilizers and subjects described in included articles}

Table 2 summarizes the number of studies that included each immobilization group. No cadaver-based studies were included. Cervico-high thoracic devices (e.g. Aspen brace, C-Breeze, Miami J, Necloc, Philadelphia, Stifneck, Vertebrace, Vista, XTW, and Yale models) were well-described in several studies [1, 6, 12-19]. None of the studies reported the effect of rigid cervical collars (type $A_{2}$ ), cranial traction (type $\mathrm{C}$ ), or halo vest (type $\mathrm{E}_{2}$ ) devices on cervical mobility.
Fig. 2 Flowchart of the study selection process

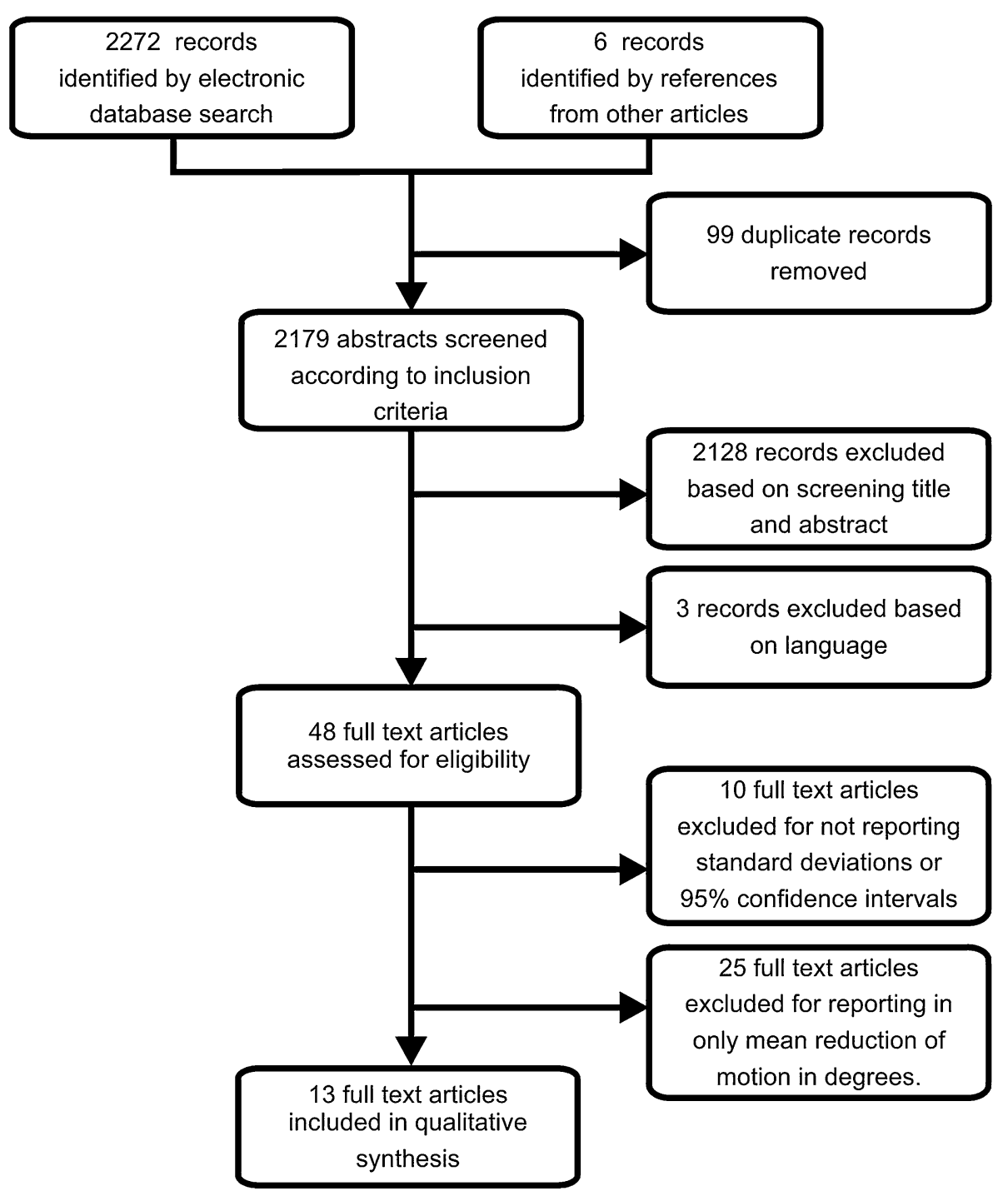


Table 1 Quality assessment summary: review authors' judgments about each quality component for each included study according to the quality assessment tool for quantitative studies (EPHPP)

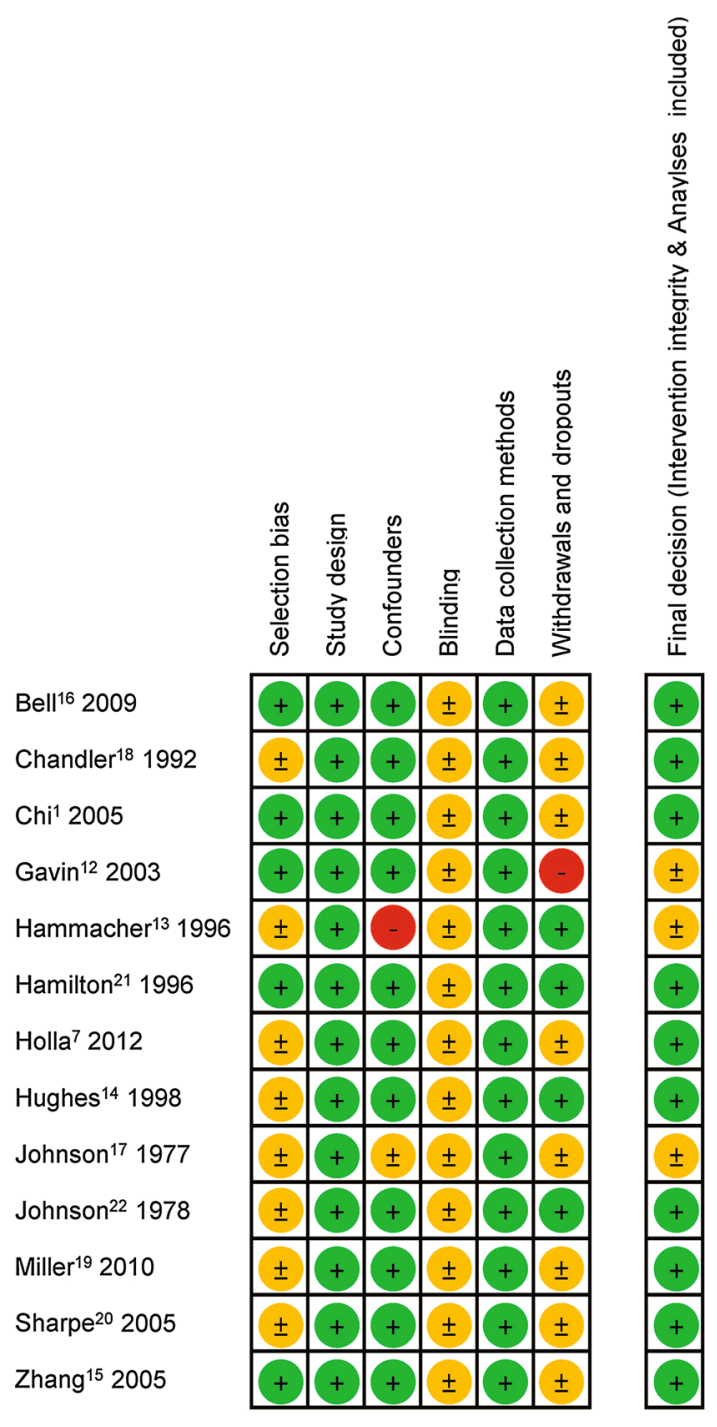

\section{The ability to restrict cervical mobility}

Table 2 and Fig. 3 summarize MRP and MIL for each device. The ability of soft collars (type $\mathrm{A}_{1}$ devices) to restrict the range of motion in all directions was poor (MIL 0-22 \%); no suitable reports for rigid collars (type $\mathrm{A}_{2}$ ) were available. The ability of cervico-high thoracic devices (type $\mathrm{B}_{1}$ ) to restrict flexion and extension was moderate to substantial (MIL 42-78\%), poor to moderate for lateral bending (MIL 13-40\%), and poor to moderate for rotation (MIL 13-40\%). Compared to other types of immobilizers, the type $\mathrm{B}_{1}$ devices had relatively high standard deviation (up to $34 \%$ ) and wide variability among studies that used the same device.

The ability of cervico-low thoracic devices (type $\mathrm{B}_{2}$ ) to restrict flexion/extension and rotation was moderate to high
(MIL 57-88 \%), whereas the ability of these devices to restrict lateral bending was poor to moderate (MIL $12-48 \%)$. None of the studies evaluated cranial traction devices (type $\mathrm{C}$ ) with respect to restricting cervical mobility. The ability of cranio-thoracic devices for non-ambulatory patients (type D) to restrict flexion, lateral bending, and rotation was substantial to nearly complete (MIL 74-92 \%), and the ability of these devices to restrict extension was moderate to nearly complete (MIL 41-84\%).

The ability of vests with non-invasive skull fixation (type $\mathrm{E}_{1}$ ) to restrict flexion and extension was substantial to nearly complete (MIL 68-90\%), nearly complete for rotation (MIL 82-98\%), and fair to nearly complete for lateral bending (MIL 32-94\%). With respect to lateral bending, only one study [20] reported a fair MIL (32\%, for the Minerva brace); the remaining studies reported MIL $\geq 70 \%$ (i.e., substantial MIL or better).

\section{Discussion}

We systematically reviewed all published articles regarding all types of external cervical immobilizers and compared their ability to restrict movement of the cervical spine. As predicted by the laws of biomechanics, the level of immobilization generally increases as both the surface area supported and the lever arm increase. Devices that only support the cervical area can restrict the normal range of motion by only $50 \%$ (or less), whereas rigid devices that provide support from cranium to the thorax provide nearly complete immobilization. Generally speaking, the classification of an external immobilizer corresponds - at least to a certain degree - to the device's ability to immobilize the cervical spine. We emphasize that the used classification is not a linear system; type $\mathrm{C}$ and type $\mathrm{D}$ immobilizers can only be applied in non-ambulatory patients.

As described by both Johnson et al. [22] and Hammacher et al. [13], the reported standard deviation of immobilization for some specific devices (e.g., soft collars, Necloc, Vertebrace, etc.) was quite high, even exceeding the mean values for immobilization. The relatively small number of participants in these studies cannot explain these large standard deviations, as high variability was reported in other, larger studies as well. In addition, the difference in the ability to immobilize the cervical spine using the same type of device varied by more than $20 \%$. Given that we corrected for differences in the normal range of motion among individuals (i.e., reporting the percentage of immobilization), any differences between individual participants do not likely explain this finding.

One explanation for the differences between studies may be the limited accuracy of the various methods used to 


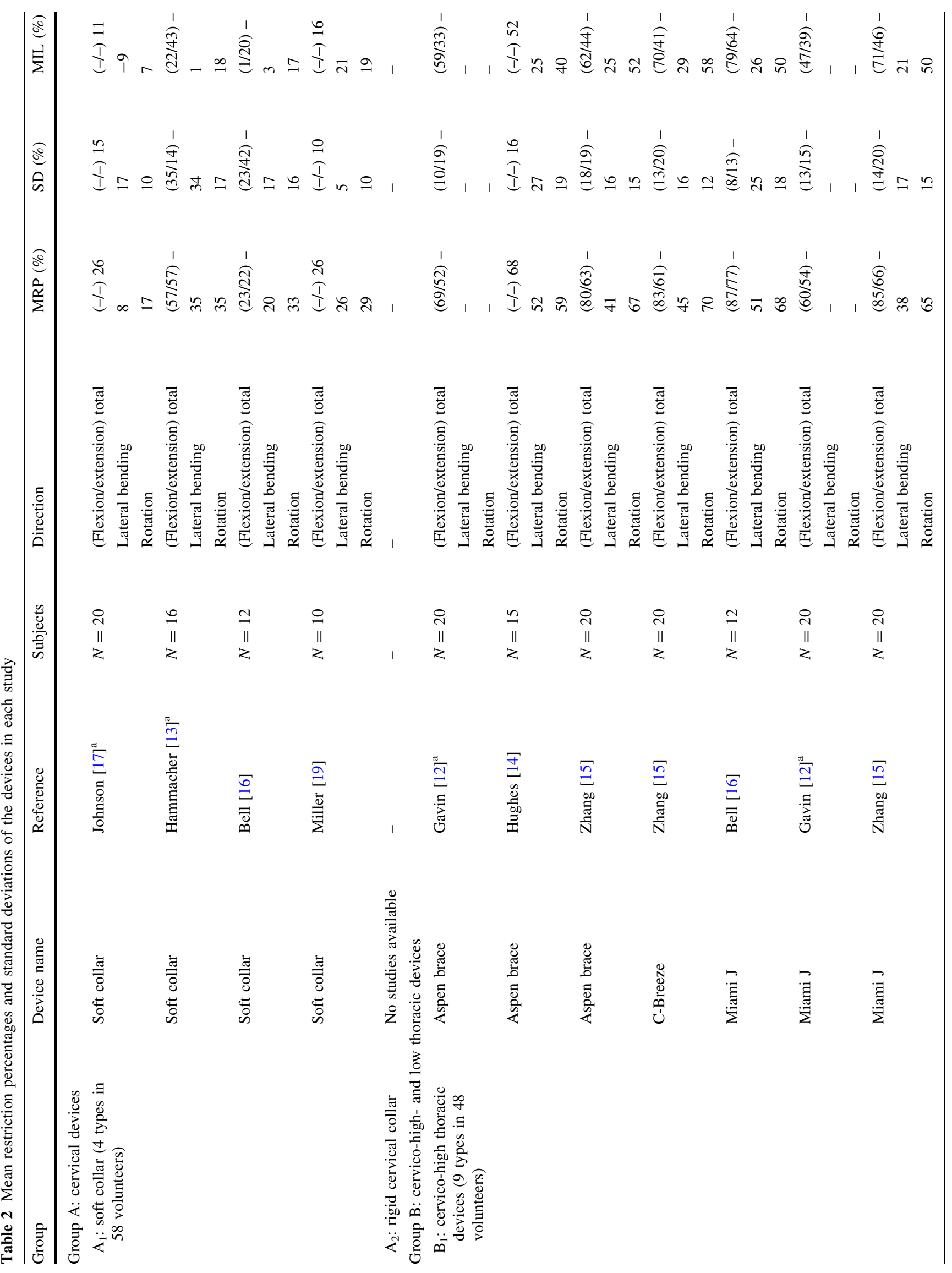




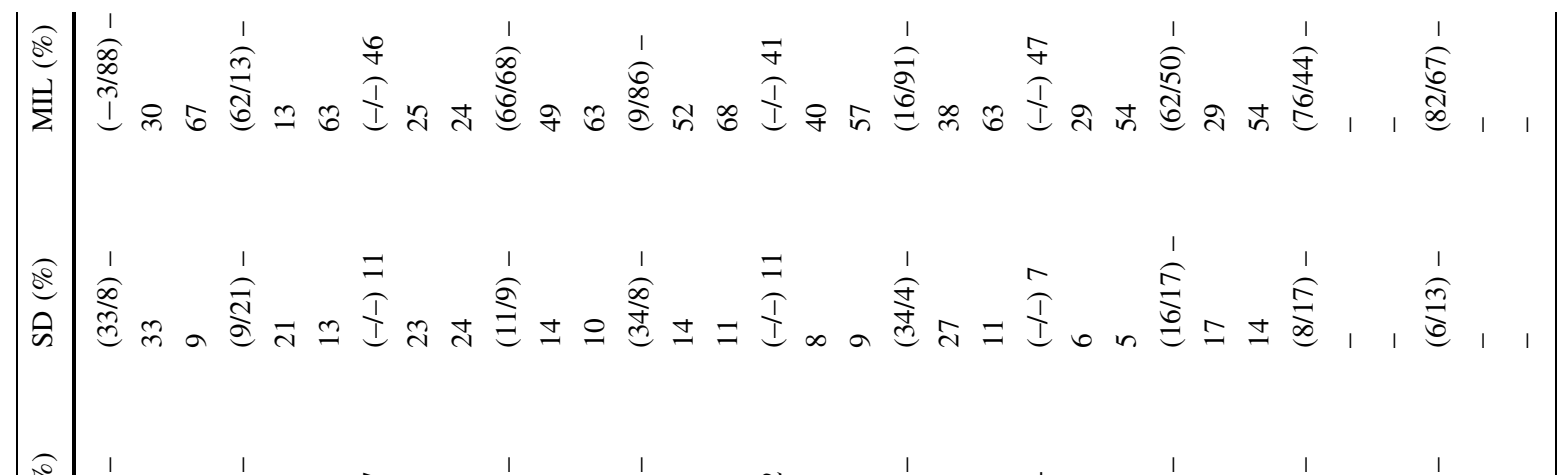

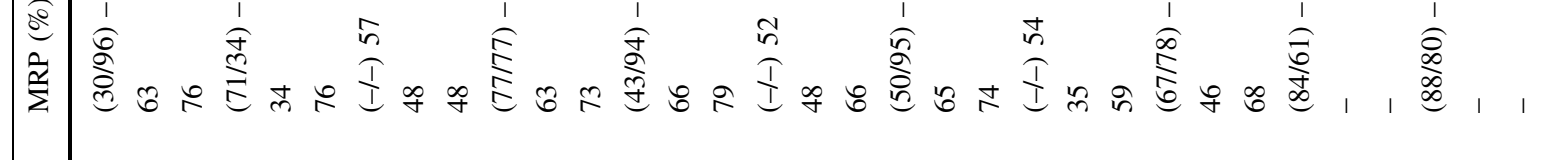

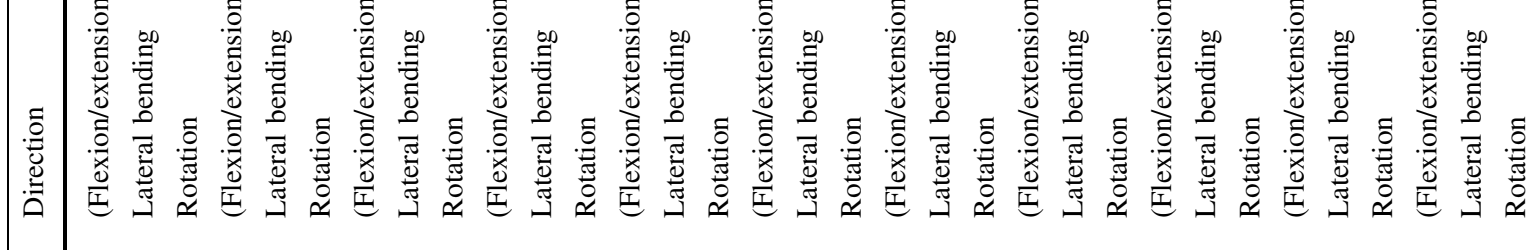

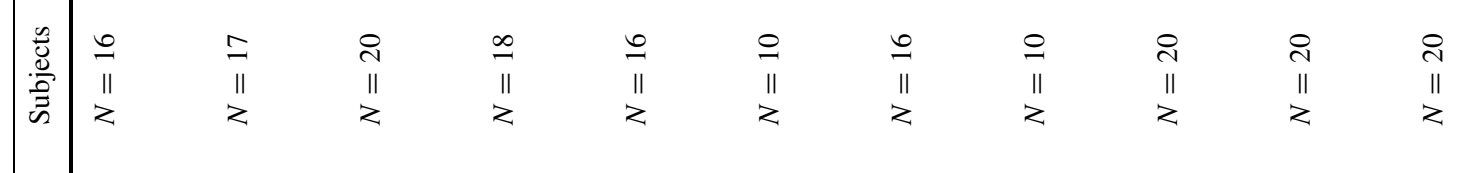

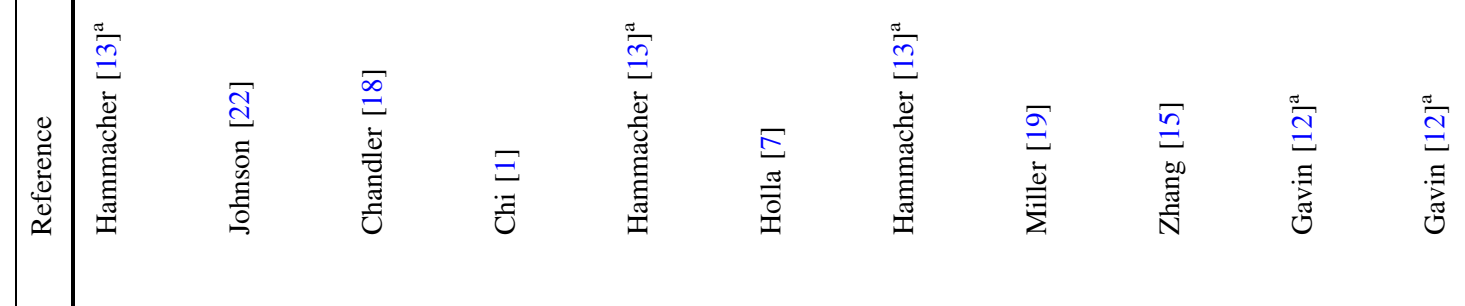

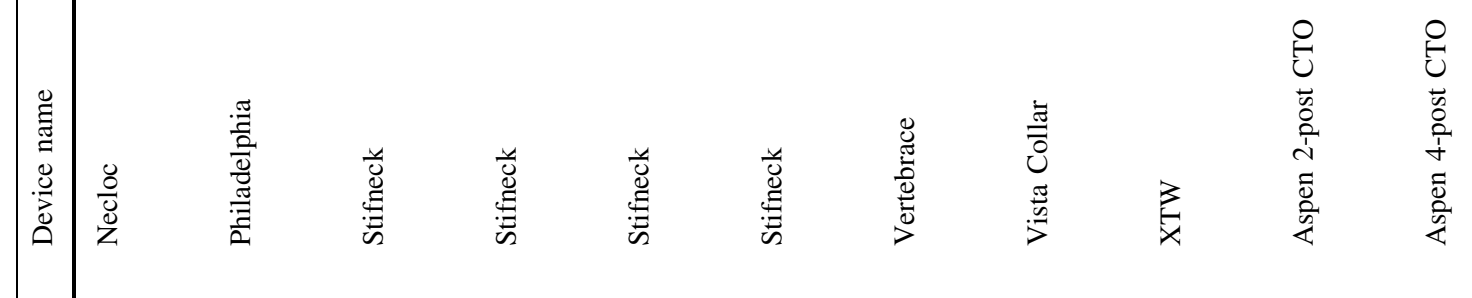

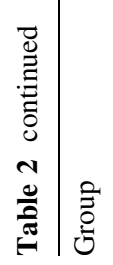

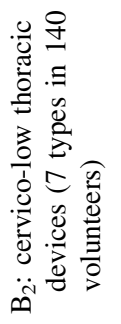




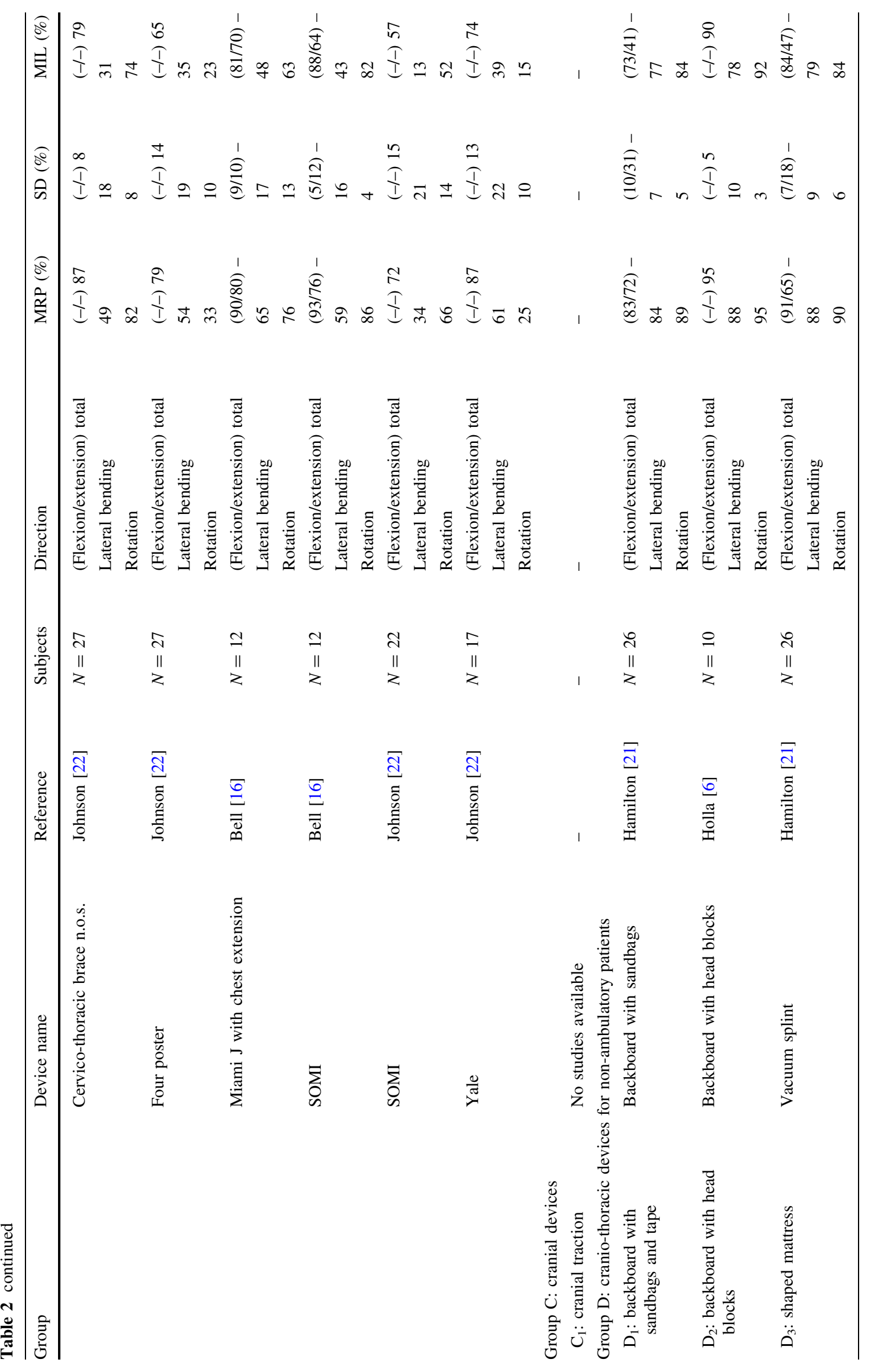




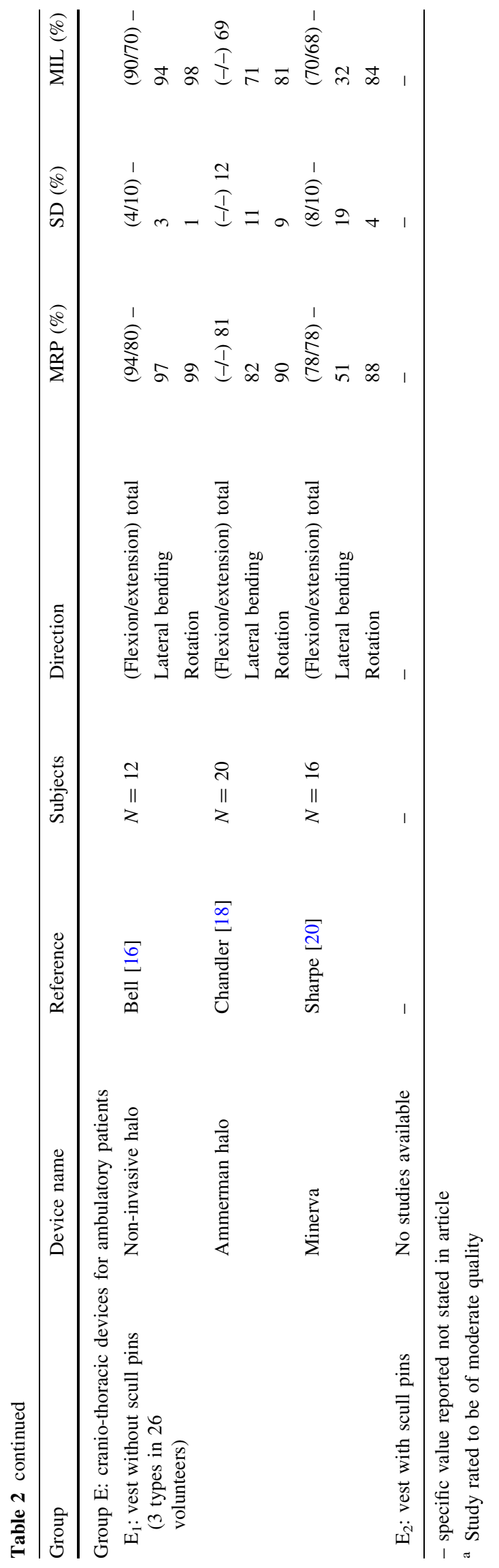

Fig. 3 a Mean restriction percentage (MRP) and minimal immobilization limit (MIL) per device in flexion and extension. Dark gray and light gray bars represent flexion and extension, respectively. In case presented percentages are identical, separate flexion and extension were not provided in the original article. Bars represent the MRP, error bars represent the MIL. b Mean restriction percentage (MRP) and minimal immobilization limit (MIL) per device for lateral bending. Bars represent the MRP, error bars represent the MIL. c Mean restriction percentage (MRP) and minimal immobilization limit (MIL) per device for rotation. Bars represent the MRP, error bars represent the MIL

measure the range of motion of the cervical spine. Another reason may lie in the different forces generated by the healthy volunteers. Applying larger forces generally results in a wider range of motion, and only experiments using cadavers enable the researcher to control the precise amount of force and correlate this force with the range of motion. However, none of the studies that met our inclusion criteria used cadavers. In addition, the size and application of the device can strongly influence its ability to restrict movement. For example, improperly placing a Stifneck collar can reduce its ability to provide immobilization by $>20 \%$ [16]. Proper sizing is also a practical issue with many external immobilizers; a cervico-thoracic device that is sized incorrectly by even a few millimeters can result in many degrees of motion in all directions. To introduce a margin of safety, we therefore developed the MIL; although this method does not entirely solve the problem of severely ill-fitting devices, it covers the usual differences between average individuals.

The ability to restrict flexion and extension was reported using several different methods. For example, some articles reported flexion and extension as separate degrees of freedom. However, this method is not ideal, as the "neutral" position of the cervical spine is unclear. A difference of only $10^{\circ}$ in the neutral position can result in a mismatch with flexion and extension by $20^{\circ}$. Some articles addressed this problem by reporting flexion and extension in one single range and one dimension. Although this eliminates the problem of the neutral head position, any separate differences in flexion and/or extension cannot be detected. In our review, both types of reports are included and described. For future research, we advise that authors report flexion and extension as two separate dimensions, and we recommend reporting flexion and extension as one single dimension.

In a 3D motion analysis study by Evans et al. [23], the effectiveness of different cervico-high thoracic immobilizers were compared to their ability to restrict spinal motion through physiological ranges. All tested immobilizers were classified as cervico-high thoracic immobilizers (type $\mathrm{B}_{1}$ : Vista, Miami-J, Miami-J advanced and Philadelphia collar). This study was not included since it was published after the performed literature search. 

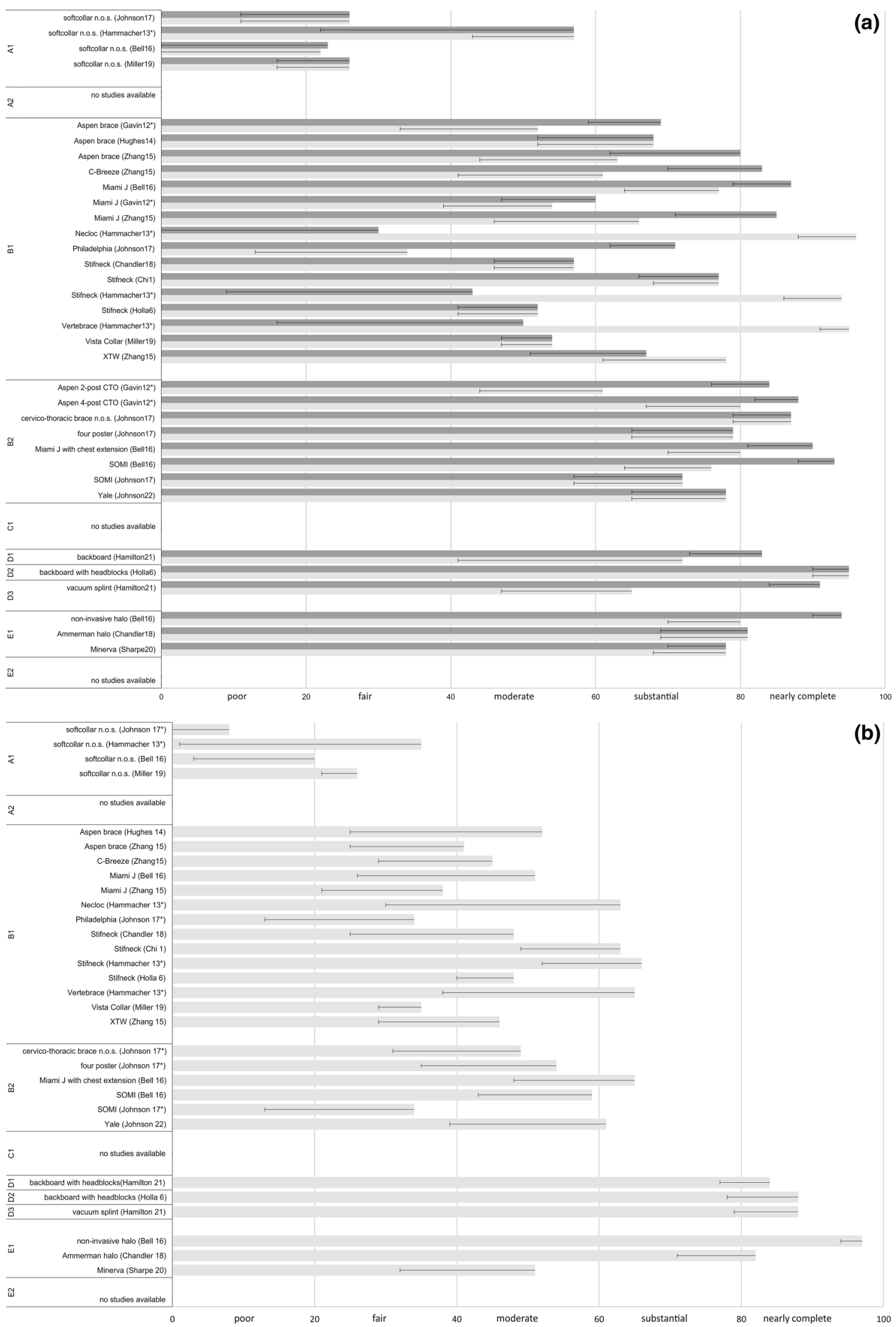


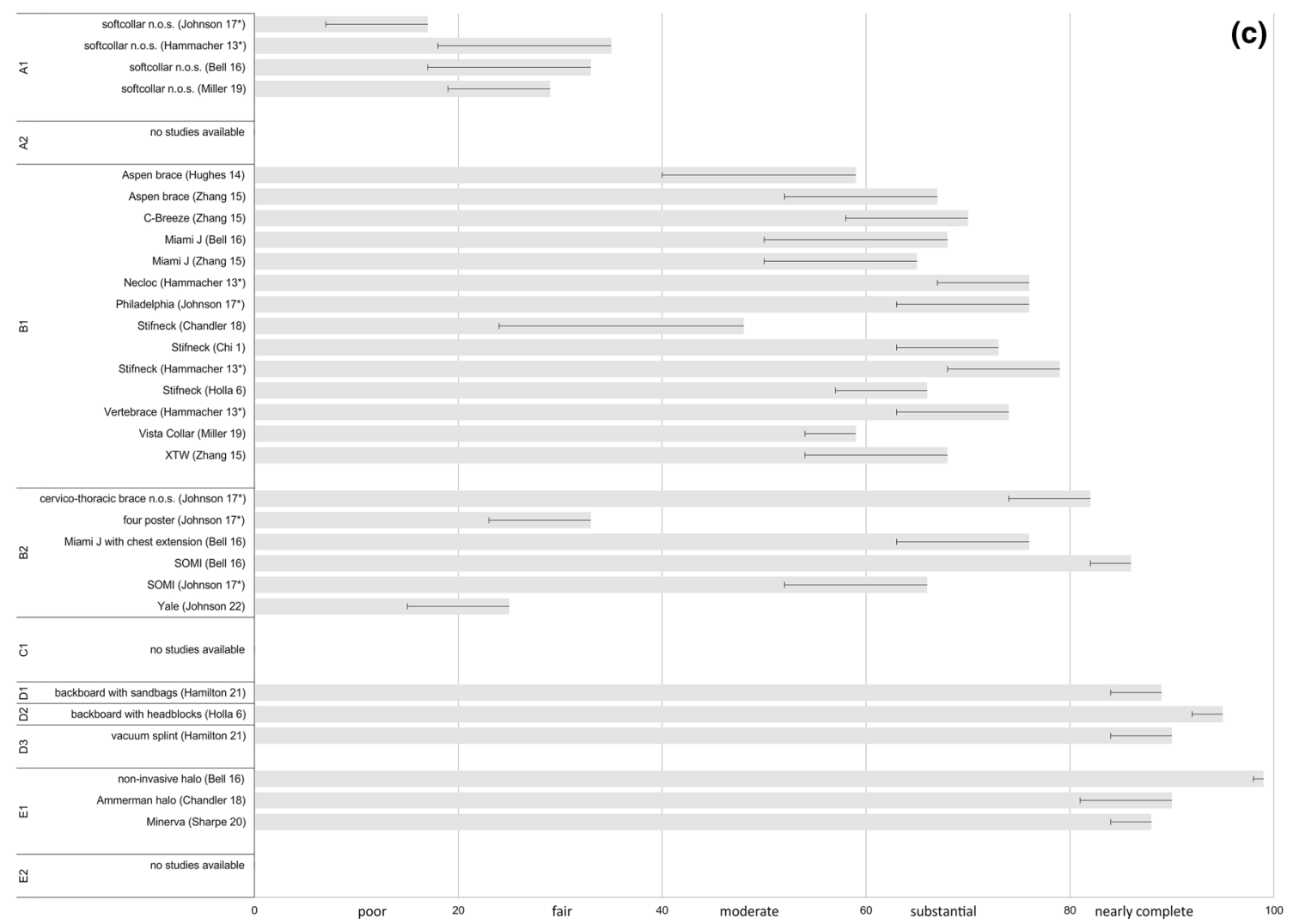

Fig. 3 continued

However, its results are in line with the results of the studies included in this systematic review; the ability to restrict flexion and extension was substantial (MIL 61-67\%) and fair to moderate for lateral bending (MIL 21-42 \%). However, Evans et al. [23] reported the ability to restrict rotation to be moderate to substantial (MIL 56-66 \%) while the studies included in this systematic review reported a poor to moderate rotational restriction (MIL 13-40\%).

To the best of our knowledge, this is the first systematic review of cervical immobilization devices based on the anatomical regions in which the devices provide support. However, some potential limitations should be discussed. First, we included only studies that reported the range of motion of healthy cervical spines. The effectiveness of an immobilizing device can potentially differ between healthy individuals and patients with a cervical spine injury. However, because including studies with various types of injuries at various cervical levels would have yielded incompatible results, we excluded such studies. Second, the MIL was used by subtracting one standard deviation from the MIL and assigned into levels of immobilization (poor, fair, moderate, substantial and nearly complete) according to pre-set percentages. These are arbitrary cut off points chosen by the authors to translate immobilization percentages into comprehensible text. However, if the mentioned cut-off percentages are increased or decreased by $5 \%$ our conclusions do not differ. Furthermore the MRP, MIL and its relation to the cut off points are clearly presented in Fig. 3. Third, this review revealed that only the total movement of the entire cervical spine is generally described. It remains unclear whether the different types of immobilizers are restricting movements at the upper or at the lower cervical spine primarily. New studies using validated techniques that can measure intervertebral movement in three dimensions are needed.

One of the most striking findings of our review is that several types of immobilizers that are currently used both widely and on a daily basis (including halo traction, halo vests, head blocks and vacuum splinting) are not described accurately in the literature. Although several reports were available with respect to cervico-thoracic devices, other groups of immobilizers completely lacked any reports or studies. This might be one of the reasons why there is no definitive evidence about the use of orthoses after spinal interventions or in painful conditions of the cervical spine [24].

In summary, this review exposes the existing gaps in our basic knowledge regarding external stabilization of the cervical spine. Therefore, researchers must investigate further the effects of current and future cervical immobilizers. Once we have sufficient insight into the ability of 
various immobilizers to restrict cervical mobility in multiple directions, practitioners can make informed choices based on scientific knowledge in order to effectively stabilize the spine for treating instability of the cervical spine.

\section{Compliance with ethical standards}

Conflict of interest No funds or grants were received in support of this work. No benefits in any form have been or will be received from a commercial party related directly or indirectly to the subject of this manuscript.

Open Access This article is distributed under the terms of the Creative Commons Attribution 4.0 International License (http://crea tivecommons.org/licenses/by/4.0/), which permits unrestricted use, distribution, and reproduction in any medium, provided you give appropriate credit to the original author(s) and the source, provide a link to the Creative Commons license, and indicate if changes were made.

\section{Appendix: Search strategy used to collect articles regarding external immobilization of the cervical spine}

The search terms used, listed by group

\begin{tabular}{|c|c|c|}
\hline Therapy group & Anatomy group & $\begin{array}{l}\text { Assessment } \\
\text { group }\end{array}$ \\
\hline Orthotic devices & Spine & Movement \\
\hline Orthotic device & $\begin{array}{l}\text { Cervical } \\
\text { vertebrae }\end{array}$ & $\begin{array}{c}\text { Range of } \\
\text { motion }\end{array}$ \\
\hline Orthoses & Cervical spine & $\begin{array}{l}\text { Head } \\
\text { movement }\end{array}$ \\
\hline Orthosis & $\begin{array}{l}\text { Cervical spine } \\
\text { injury }\end{array}$ & Immobilisation \\
\hline Orthopedic equipment & Cervicothoracic & Immobilization \\
\hline Collar & Cranio-thoracic & Biomechanics \\
\hline Soft collar & Neck & Rotation \\
\hline Semi-rigid collar & $\begin{array}{l}\text { Atlantoaxial } \\
\text { joint }\end{array}$ & Kinetics \\
\hline Rigid collar & $\begin{array}{l}\text { Atlanto-occipital } \\
\text { joint }\end{array}$ & \\
\hline \multicolumn{3}{|l|}{ Braces } \\
\hline \multicolumn{3}{|l|}{ Traction } \\
\hline \multicolumn{3}{|l|}{ Sandbags } \\
\hline \multicolumn{3}{|l|}{ Head blocks } \\
\hline \multicolumn{3}{|l|}{ Spine board } \\
\hline \multicolumn{3}{|l|}{ Backboard } \\
\hline \multicolumn{3}{|l|}{ Vacuum mattress } \\
\hline \multicolumn{3}{|l|}{ Surgical casts } \\
\hline \multicolumn{3}{|l|}{ Casts } \\
\hline \multicolumn{3}{|l|}{ Minerva } \\
\hline Noninvasive halo vest & & \\
\hline
\end{tabular}

Therapy group Anatomy group Assessment group

Noninvasive halovest

CTO

Cervicothoracic orthoses

Cervicothoracic orthosis

SOMI

Sternal-occipital-mandibularimmobilizer

Halo vest

Halo vest

Halo

\section{Search strings used in this article for search in MEDLINE}

The following groups were combined using the string: "A" AND "B" AND "C".

\section{Therapy (A)}

"Orthotic devices" [MeSH Terms] OR "orthotic devices"[Text Word] OR "orthotic device"[Text Word] OR "orthosis"[Text Word] OR "orthoses"[Text Word] OR "collar"[Text Word] OR "soft collar"[Text Word] OR "semi-rigid collar" [Text Word] OR "rigid collar"[Text Word] OR "braces"[MeSH Terms] OR "brace"[Text Word] OR "traction" [MeSH Terms] OR traction[Text Word] OR sandbags[Text Word] OR "head blocks"[Text Word] OR "spine board" [Text Word] OR "backboard"[Text Word] OR "vacuum mattress"[Text Word] OR “casts, surgical" [MeSH Terms] OR "cast"[Text Word] OR minerva[Text Word] OR "noninvasive halo vest"[Text Word] OR "noninvasive halovest"[Text Word] OR CTO[Text Word] OR "cervicothoracic orthoses" [Text Word] OR "cervicothoracic orthosis"[Text Word] OR "somi" [Text Word] OR sternal-occipital-mandibular-immobilizer[Text Word] OR "halo vest"[Text Word] OR halo[Text Word] OR "halovest"[Text Word].

\section{Anatomy group (B)}

"Spine"[MeSH Terms] OR "spine"[Text Word] OR "cervical vertebrae"[MeSH Terms] OR cervical vertebrae[Text Word] OR "cervical spine" [Text Word] OR "cervical spine injury"[Text Word] OR "cervicothoracic"[Text Word] OR "cranio thoracic"[Text Word] OR "neck" [MeSH Terms] OR neck[Text Word] OR "atlantoaxial joint"[Text Word] OR "atlanto occipital 
joint"[Text Word] OR “atlanto axial joint"[MeSH Terms] OR "atlanto occipital joint”[MeSH Terms].

\section{Assessment group $(C)$}

"Movement"[MeSH Terms] OR "movement"[Text Word] OR "range of motion, articular" [MeSH Terms] OR "range of motion" [Text Word] OR "head movements"[MeSH Terms]) OR "head movement"[Text Word] OR "immobilisation" [MeSH Terms] OR "immobilisation"[Text Word] OR “immobilisation"[Text Word] OR "biomechanics"[MeSH Terms] OR "biomechanics"[Text Word] OR "rotation" [MeSH Terms] OR "rotation"[Text Word] OR "kinetics"[MeSH Terms] OR "kinetics"[Text Word].

\section{Search strings used in this article for search in EMBASE, CENTRAL and the CBRG trials}

The following groups were combined using the string: " $A$ " AND "B" AND "C".

\section{Therapy group (A)}

"Orthotic devices" OR "orthotic device" OR "orthoses" OR "orthosis" OR "orthopedic equipment" " OR "collar" OR "soft collar" OR "semi-rigid collar" OR "rigid collar" OR "braces" OR "traction" OR "sandbags" OR "head blocks" OR "spine board" OR "backboard" OR "vacuum mattress" OR "surgical casts" OR "cast" OR "minerva" OR "noninvasive halo vest" " OR "noninvasive halovest" OR "CTO" OR "cervicothoracic orthoses" OR "SOMI" OR "sternal-occipital-mandibular-immobilizer" OR "halo vest" OR "halovest" OR "halo".

\{Including Related Terms .

Anatomy group (B)

"Spine" OR “cervical vertebrae" OR "cervical spine" OR "cervicothoracic" OR "neck" OR "atlantoaxial joint" OR "atlanto-occipital joint" OR "cervical spine injury" OR "cranio-thoracic".

\{Including Related Terms \}.

\section{Assessment group (C)}

"Movement" OR "range of motion" OR "head movement" OR "immobilisation" OR "immobilisation" OR "biomechanics" OR "kinetics".

\{Including Related Terms\}.

\section{References}

1. Chi CH, Wu FG, Tsai SH, Wang CH, Stern SA (2005) Effect of hair and clothing on neck immobilization using a cervical collar. Am J Emerg Med 23(3):386-390

2. Frohna WJ (1999) Emergency department evaluation and treatment of the neck and cervical spine injuries. Emerg Med Clin North Am 17(4):739-791

3. Hadley N, Wlters C, Grabb PA, Oyeskiu NM, Przybylski GJ, Resnick DK, Ryken TC (2001) Guidelines for the management of acute cervical spine and spinal cord/section of disorders of the spine and peripheral nerves, 2001 [American Association Neuro Surgeons web site]. http://www.aans.org/ /media/Files/Educa tion. Accessed Oct 2014

4. Kwan I, Bunn F, Roberts I (2001) Spinal immobilisation for trauma patients. Cochrane Database Syst Rev 2:CD002803

5. Kwan I, Bunn F (2005) Effects of prehospital spinal immobilization: a systematic review of randomized trials on healthy subjects. Prehosp Disaster Med 20(1):47-53

6. Holla M (2012) Value of a rigid collar in addition to head blocks: a proof of principle study. Emerg Med J 29(2):104-107

7. Holla M, Huisman JMR, Hosman AJF (2013) A validated classification for external immobilization of the cervical spine. Evid Based Spine Care J 04(02):072-077

8. Deeks JJ, Dinnes J, D'Amico R, Sowden AJ, Sakarovitch C, Song F, Petticrew M, Altman DG (2003) Evaluating non-randomised intervention studies. Health Technol Assess 7(27):iii-x, 1-173

9. Williams MA, McCarthy CJ, Chorti A, Cooke MW, Gates S (2010) A systematic review of reliability and validity studies of methods for measuring active and passive cervical range of motion. J Manip Physiol Ther 33(2):138-155

10. Higgins J, Green S (2011) Cochrane handbook for systematic reviews of interventions [The Cochrane Collaboration website]. Version 5.1.0 updated March 2011. http://handbook.cochrane. org. Accessed Oct 2014

11. Gravetter FJ. Wallnau LB (2008) Essentials of statistics for the behavioral sciences Thomson Learning Inc.

12. Gavin TM, Carandang G, Havey R, Flanagan P, Ghanayem A, Patwardhan AG (2003) Biomechanical analysis of cervical orthoses in flexion and extension: a comparison of cervical collars and cervical thoracic orthoses. J Rehabil Res Dev 40(6):527-537

13. Hammacher ER, Schouwerwou RJ, Lichtveld RA, van der Werken C (1996) Een vergelijkend onderzoek naar de effectiviteit van vier halskragen. Ned Tijdschr Traum 4(1):6-10 (article in Dutch)

14. Hughes SJ (1998) How effective is the Newport/Aspen collar? A prospective radiographic evaluation in healthy adult volunteers. J Trauma 45(2):374-378

15. Zhang S, Wortley M, Clowers K (2005) Evaluation of efficacy and 3D kinematic characteristics of cervical orthoses. Clin Biomech (Bristol, Avon) 20(3):264-269

16. Bell KM, Frazier EC, Shively CM, Hartman RA, Ulibarri JC, Lee JY, Kang JD, Donaldson WF 3rd (2009) Assessing range of motion to evaluate the adverse effects of ill-fitting cervical orthoses. Spine J 9(3):225-231

17. Johnson RM, Hart DL, Simmons EF, Ramsby GR, Southwick WO (1977) Cervical orthoses. A study comparing their effectiveness in restricting cervical motion in normal subjects. J Bone Joint Surg Am 59(3):332-339

18. Chandler DR, Nemejc C, Adkins RH, Waters RL (1992) Emergency cervical spine immobilization. Ann Emerg Med 21(10):1185-1188 
19. Miller CP, Bible JE, Jegede KA, Whang G, Grauer N (2010) Soft and rigid collars provide similar restriction in cervical range of motion during fifteen activities of daily living. Spine (Phila Pa 1976) 35(13):1271-1278

20. Sharpe KP, Rao S, Ziogas A (1995) Evaluation of the effectiveness of the Minerva cervicothoracic orthosis. Spine (Phila Pa 1976) 20(13):1475-1479

21. Hamilton RS, Pons PT (1996) The efficacy and comfort of fullbody vacuum splints for cervical-spine immobilization. J Emerg Med 14(5):553-559

22. Johnson RM, Hart DL, Owen JR, Lerner E, Chapin W, Zeleznik $R$ (1978) The Yale cervical orthosis: an evaluation of its effectiveness in restricting cervical motion in normal subjects and a comparison with other cervical orthoses. Phys Ther 58(7):865-871

23. Evans NR, Hooper G, Edwards R, Whatling G, Sparkes V, Holt C, Ahuja S (2013) A 3D motion analysis study comparing the effectiveness of cervical spine orthoses at restricting spinal motion through physiological ranges. Eur Spine J 22(Suppl 1):S10-S15

24. Zarghooni K, Beyer F, Siewe J, Eysel P (2013) The orthotic treatment of acute and chronic disease of the cervical and lumbar spine. Dtsch Arztebl Int 110(44):737-742 